\title{
Double-mode Chaos with Hard Nonlinearities Coupled with an Inductor
}

\author{
Masaaki Kojima $^{\dagger}+$, Yoko Uwate ${ }^{\dagger}$ and Yoshifumi Nishio ${ }^{\dagger}$ \\ $\dagger$ Department of Electrical and Electronic Engineering, Tokushima University \\ 2-1 Minami-Josanjima, Tokushima, 770-8506, Japan \\ \$Engineering Administration Department, NHK \\ 2-2-1 Jinnan, Shibuya-ku, Tokyo, 150-8001, Japan \\ Email: kojima, uwate, nishio@ee.tokushima-u.ac.jp
}

\begin{abstract}
We propose two chaotic circuits with hard nonlinearities coupled with an inductor and investigate some interesting phenomena in these systems. At first, we look into that several types of synchronization modes are stably excited. Next, we confirm that the synchronization modes are chaotically vibrated by observation of attractors and Poincare maps in the circuit systems. In particular, we aim to find double-mode oscillations with two different types of chaotic phenomena in these systems.
\end{abstract}

\section{Introduction}

Coupled oscillator systems with nonlinear characteristics have shown some interesting phenomena and brought improvement to natural science and fundamental research. It is known that two oscillators coupled with an inductor excite two different modes: In-phase single-mode and anti-phase single-mode coexist for some range of parameter values. Both of negative resistors within these oscillators are normal. Such an oscillator is called a soft oscillator or one with soft nonlinearity. In comparison with soft oscillator, hard oscillator has a nonlinear negative resistor whose $i-v$ characteristics contain both negative and positive. The characteristics near the origin act as a positive resistor. Therefore the oscillator converges on the origin for small initial values. Greater initial values than the origin stable regions are required to generate the oscillation. Such an oscillator is called hard oscillator or one with hard nonlinearities. Two oscillators with hard nonlinearities coupled with an inductor excite four different modes: Zero (non-oscillation), in-phase single-mode, anti-phase single-mode and double-mode coexist for some range of parameter values [1]. The double-mode shows simultaneous asynchronous oscillations with in-phase and anti-phase frequency components. In particular, the double-mode oscillations are stably excited although they do not occur in the case of common negative resistors with soft nonlinearities.

On the basis of this study, we have proposed two coupled chaotic circuits with hard nonlinearities for the purpose of looking for the interesting phenomena as double-mode chaotic oscillations [2][3]. We have confirmed doublemode chaos with one-periodic in-phase and chaotic antiphase single-modes [2] and that with torus and chaos [3], respectively. However, we have not found that with chaos and chaos in these systems. We are considering the reason that phenomena do not exist could be caused by their single circuit architecture. Bifurcation phenomena to chaos depend on the circuit architecture.

In this study, we propose two chaotic circuits with hard nonlinearities coupled with an inductor and look for the expected double-mode chaos. Especially, we have adopted a different type of single circuit from the previous studies [2][3].

\section{Circuit Model}

Figure 1 shows the proposed circuit model. Two chaotic circuits with hard nonlinearities are coupled with an inductor in the model. Each single circuit consists of an capacitor, inductors, diodes and nonlinear negative resistors. The i-v characteristics of the diodes are shown in Fig 2.

$$
v_{d}(i)=\left\{\begin{array}{lll}
r_{d} i & \cdots & \left(i \leqq V / r_{d}\right) \\
V & \cdots & \left(i>V / r_{d}\right)
\end{array}\right.
$$

On the basis of the characteristics of the diodes, the nonlinear negative resistors and fourteen diodes can be shown in Figs 3 and 4, respectively.

The number of series-connected diodes is proportional to amplitude of each oscillation. Six diodes were adopted in the previous studies [2][3]. An attractor in this model is more dented than the previous model [2][3] though each

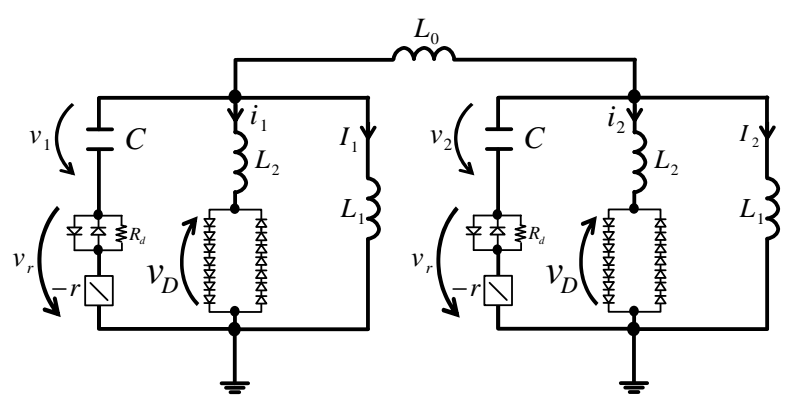

Figure 1: Circuit model. 


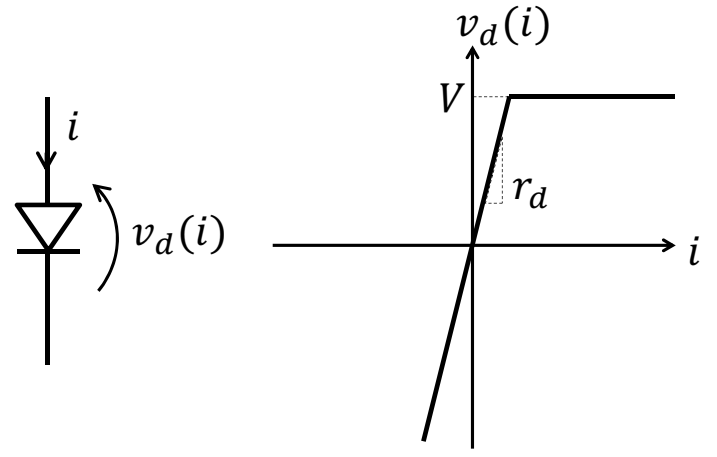

Figure 2: $i-v$ characteristics of a diode.

hard oscillator has to avoid exciting on the origin stable regions as the positive resistor. Therefore the fourteen diodes have been adopted in this model.

$$
\begin{aligned}
& v_{r}(i)=\left\{\begin{array}{l}
V-r i \cdots(i>J) \\
\left(\frac{R_{d} r_{d}}{2 R_{d}+r_{d}}-r\right) i \quad \cdots \quad(|i| \leqq J) \\
-V-r i \cdots \quad(i<-J),
\end{array}\right. \\
& \text { (where } \left.J=\frac{2 R_{d}+r_{d}}{R_{d} r_{d}} V\right) \text {, }
\end{aligned}
$$

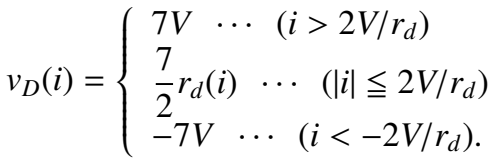

The equations governing the circuit in Fig. 1 are described as follows:

$\left.L_{1} \frac{d I_{k}}{d t}=-v_{r}+\left(I_{k}+i_{k}-\frac{L_{1}}{L_{0}}\left(I_{2}-I_{1}\right) \cdot(-1)^{k+1}\right)-v_{k}\right)$ $L_{2} \frac{d i_{k}}{d t}=-v_{r}+\left(I_{k}+i_{k}-\frac{L_{1}}{L_{0}}\left(I_{2}-I_{1}\right) \cdot(-1)^{k+1}\right)-v_{k}-v_{D}\left(i_{k}\right)$ $C \frac{v_{k}}{d t}=I_{k}+i_{k}-\frac{L_{1}}{L_{0}}\left(I_{2}-I_{1}\right) \cdot(-1)^{k+1}$

$(k=1,2)$

By changing the variables and parameters,

$$
\begin{aligned}
& I_{k}=\sqrt{\frac{C}{L_{1}}} E x_{k}, \quad i_{k}=\sqrt{\frac{C}{L_{1}}} E y_{k}, \quad v_{k}=E z_{k}, \\
& t=\sqrt{L_{1} C} \tau, \quad, . "=\frac{d}{d \tau} \\
& \alpha=\frac{L_{1}}{L_{2}}, \quad \beta=r \sqrt{\frac{C}{L_{1}}}, \quad \gamma=\frac{L_{1}}{L_{0}}, \\
& a=\frac{R_{d} r_{d}}{2 R_{d}+r_{d}} \sqrt{\frac{C}{L_{1}}}, \quad b=\frac{r_{d}}{2} \sqrt{\frac{C}{L_{1}}} . \\
& (k=1,2)
\end{aligned}
$$

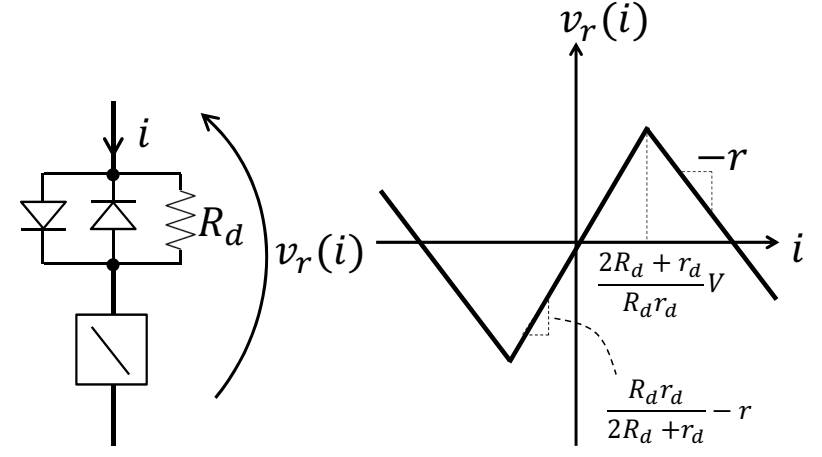

Figure 3: $i-v$ characteristics of a negative resistor.

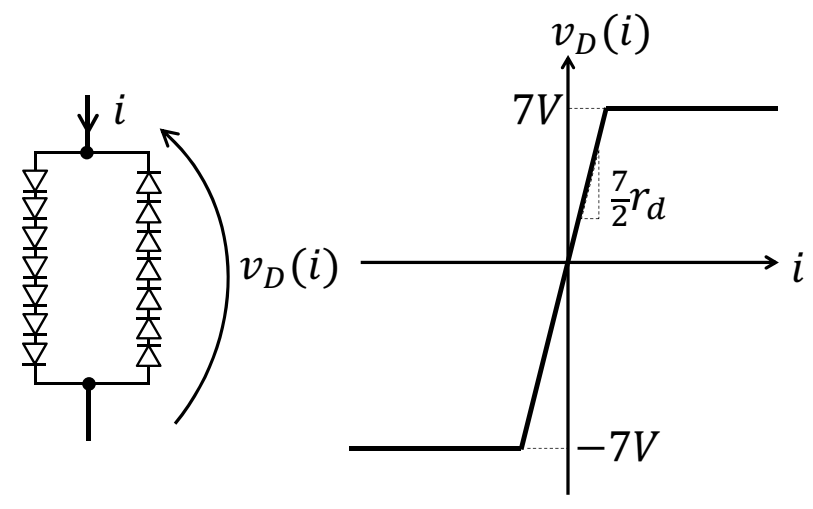

Figure 4: $i-v$ characteristics of a negative resistor.

(1) is normalized as follows:

$$
\begin{aligned}
& \dot{x}_{k}=-f_{r}\left(x_{k}+y_{k}-\gamma\left(x_{2}-x_{1}\right) \cdot(-1)^{k+1}\right)-z_{k} \\
& \dot{y}_{k}=\alpha\left\{-f_{r}\left(x_{k}+y_{k}-\gamma\left(x_{2}-x_{1}\right) \cdot(-1)^{k+1}\right)-z_{k}-f_{D}\left(y_{k}\right)\right\} \\
& \dot{z}_{k}=x_{k}+y_{k}-\gamma\left(x_{2}-x_{1}\right) \cdot(-1)^{k+1} \\
& (k=1,2)
\end{aligned}
$$

The functions $f_{r}$ and $f_{D}$ correspond to $v_{r}$ (nonlinear negative resistor) and $v_{D}$ (fourteen diodes), respectively, and are represented as follows:

$$
\begin{gathered}
f_{r}(x)=\left\{\begin{array}{ccc}
1-\gamma x & \cdots & (x>1 / a) \\
(a-\gamma) x & \cdots & (|x| \leqq 1 / a) \\
-1-\gamma x & \cdots & (x<-1 / a),
\end{array}\right. \\
f_{D}(x)=\left\{\begin{array}{ccc}
7 & \cdots & (x>1 / b) \\
7 b x & \cdots & (|i| \leqq 1 / b) \\
-7 & \cdots & (x<-1 / b) .
\end{array}\right.
\end{gathered}
$$

\section{Multi-mode Chaos}

Four different oscillation modes (zero, in-phase singlemode, anti-phase single-mode and double-mode) coexist in the circuit model of the Fig. 1. 

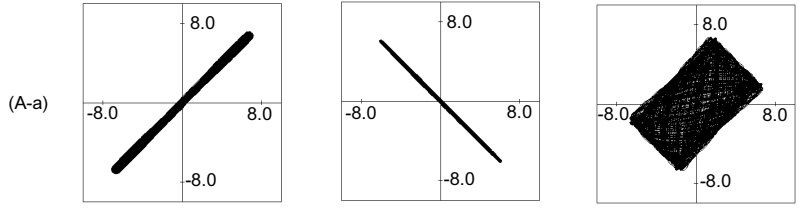

(A-b)
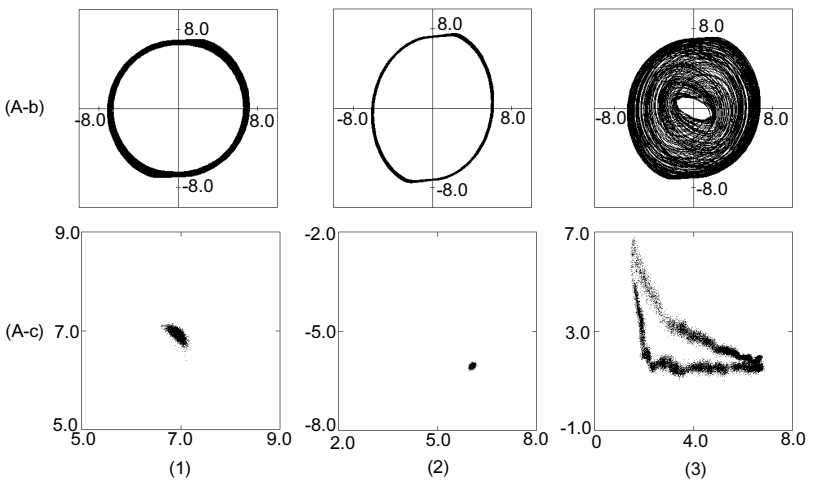

$A: \beta=0.175$
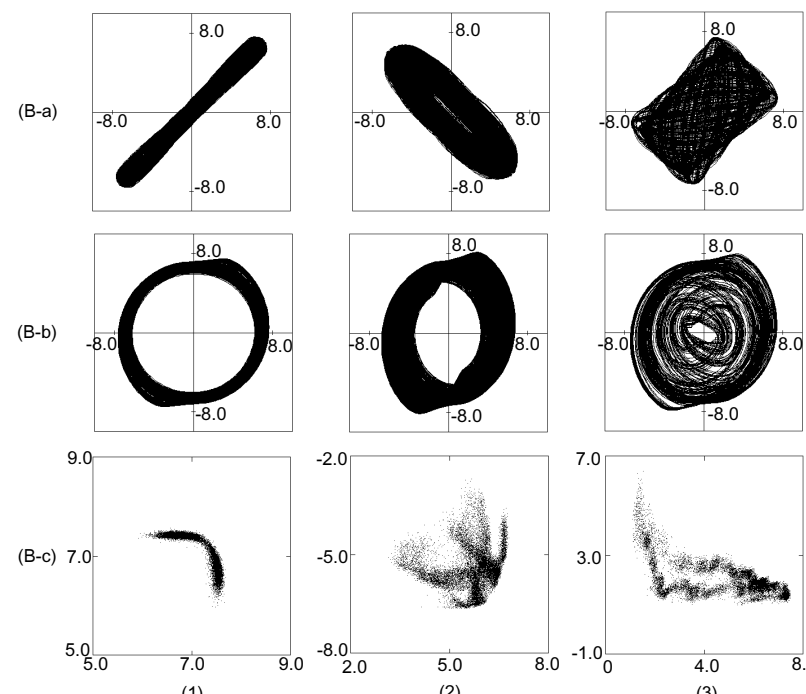

$\beta=0.2$

Figure 5: Attractors and Poincare maps of three different oscillation modes. (a) $x_{1}-x_{2}$ Attractors. (b) $x_{1}-z_{1}$ Attractors. (c) $x_{1}$ - $x_{2}$ Poincare maps. (A) $\beta=0.175$. (B) $\beta=0.2$. (1) In-phase single-mode. (2) Anti-phase single-mode. (3) Double-mode.

Each mode is determined by the initial values. The initial values $\left(x_{1}, x_{2}, y_{1}, y_{2}, z_{1}, z_{2}\right)$ of in-phase singlemode, anti-phase single-mode and double-mode are $(6.3,6.5,1.8,1.5,6.4,6.5), \quad(4.0,-4.1,0.6,-0.5,4.4,-4.2)$ and $(4.0,5.9,1.6,-1.5,-5.9,6.0)$, respectively (Figs. 5 and $6)$. In this section, we show some simulation results of some attractors and Poincare maps on each mode to investigate its behavior when the parameter $\beta$ as the absolute values of the negative resistor increases (Fig. 5). The other parameters are fixed as $\alpha=26.5, \gamma=0.28, a=1.15, b=$ 40 (Figs. 5 and 6).
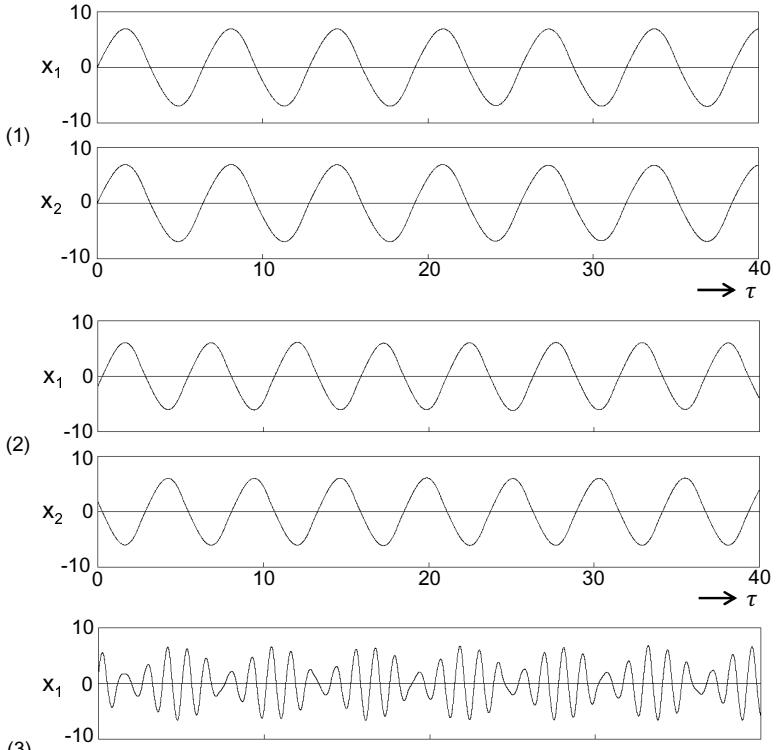

(3)
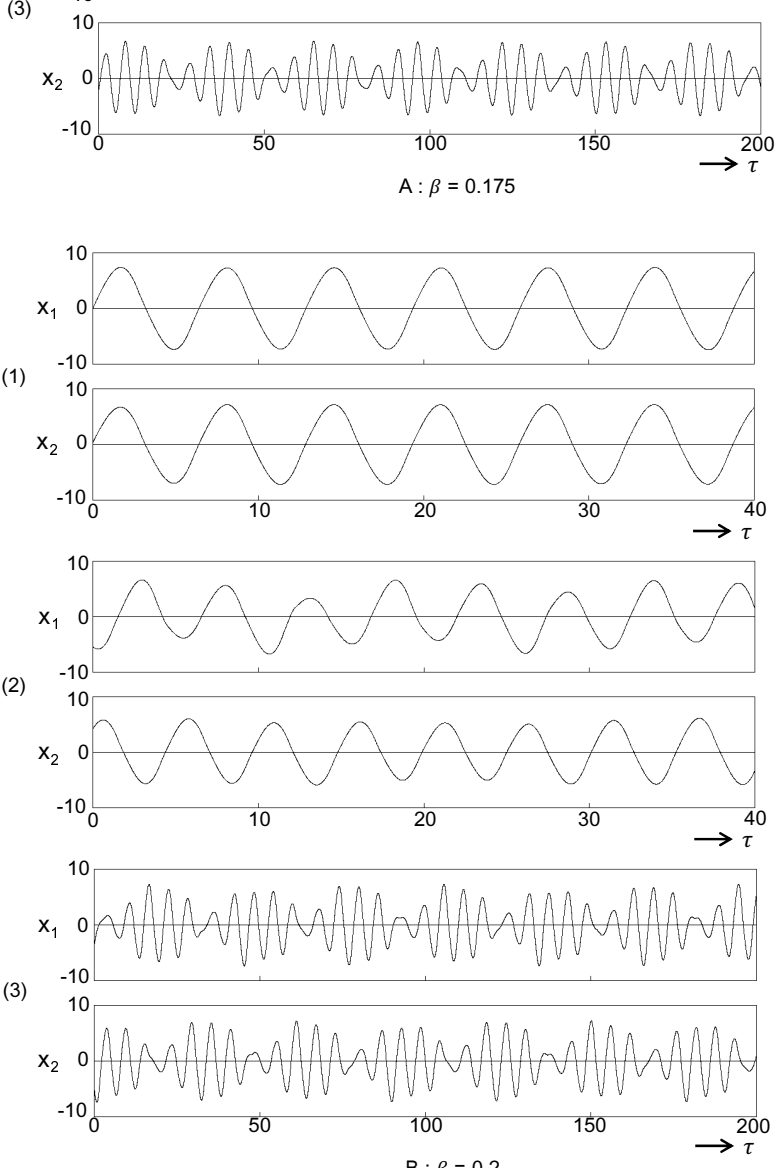

Figure 6: Time waveforms vs amplitude of three different oscillation modes. (A) $\beta=0.175$. (B) $\beta=0.2$. (1) In-phase single-mode. (2) Anti-phase single-mode. (3) Double-mode.

Zero means that neither of the two oscillators is excited. This state is always stable in some range of small initial values. We do not mention this state in the rest of this manuscript because it is trivial. 
In-phase single-mode means that the two oscillators are synchronized with the in-phase. Although they are exactly the quasi-in-phase synchronization, we see it as the inphase in this study. The in-phase single-mode is chaotic oscillation at $\beta=0.175$ and 0.2 by the attractors and Poincare maps. When $\beta$ increases, we can see that the in-phase single-mode is more chaotic.

Anti-phase single-mode means that the two oscillators are synchronized with $\pi$ phase difference. Although they are not synchronized with exact $\pi$ phase difference, we also see it as the anti-phase in this study. The anti-phase singlemode is chaotic oscillation at $\beta=0.175$ and 0.2 by the attractors and Poincare maps. When $\beta$ increases, we can see that the anti-phase single-mode is more chaotic.

Double-mode means that the above two single-mode oscillations (in-phase and anti-phase) are excited, simultaneously and asynchronously. The double-mode is chaotic oscillation at $\beta=0.175$ and 0.2 by the attractors and Poincare maps. When $\beta$ increases, we can see that the Poincare maps of the double-mode is more chaotic because the Poincare maps more spread out and breakdown.

Figure 6 shows time waveforms of three different oscillation modes. The frequency of the anti-phase single-mode is higher than that of the in-phase. The envelope periods of the double-mode time waveforms show the frequency differences between the in-phase and anti-phase singlemodes. When $\beta$ increases, we can also see that the three modes are more chaotic from the time waveforms.

\section{Conclusions}

We proposed two identical chaotic circuits with hard nonlinearities coupled with an inductor and investigated the double-mode chaos in these systems by looking at some attractors and Poincare maps. As a result, we confirmed the interesting phenomena as double-mode chaos with chaos (in-phase) and chaos (anti-phase) by computer simulations. We will analyze the bifurcation of these three modes in the near future.

\section{References}

[1] S. P. Datardina and D. A. Linkens, "Multimode oscillations in mutually coupled van der Pol type oscillators with fifth-power nonlinear characteristics," IEEE Trans. Circuits Syst., vol. 25, no. 5, pp. 308-315, May 1978.

[2] Y. Nishio and A. Ushida, "Multimode chaos in two coupled chaotic oscillators with hard nonlinearities," IEICE Trans. Fundamentals, vol. E79-A, no. 2, pp. 227-232, Feb. 1996.

[3] M. Kojima, M. Yamauchi, Y. Nishio and A. Ushida, "Simultaneous asynchronous oscillation of chaos and torus in coupled chaotic circuits," Proc. of NOLTA '00, vol. 2, pp. 567-570, Sep. 2000. 